\title{
CRÍTICA DE LA CRÍTICA CRÍTICA. CONTRA ENRIQUE HABA Y CONSORTES *
}

\author{
Manuel Atienza \\ Universidad de Alicante
}

RESUMEN. Después de señalar los elementos fundamentales de la crítica que HABA dirige a la teoría «estándar» de la argumentación jurídica, el autor entiende que «HABA tiene alguna razón en lo que dice, pero no en cómo lo dice ni tampoco (sobre todo) en la consecuencia que pretende extraer de su crítica: la descalificación radical de la teoría estándar de la argumentación jurídica». Aclara que, en su opinión, esa teoría es susceptible de más de una crítica pero que, en su opinión, HABA «ni siquiera ha planteado sus críticas con el rigor exigible para ser sometidas a una discusión racional», y termina pidiéndole que lo haga.

Palabras clave: argumentación jurídica, E. P. HABA, M. AtienZA.

ABSTRACT. After pointing out the main elements of the criticism which HABA addresses at the "standard» theory of legal argumentation, the author considers that "HABA is right to a certain extent in what he says, but not in how he says it nor (and above all) in what he considers to be the consequence of his criticism: the radical disqualification of the standard theory of legal argumentation"». The author explains that, in his opinion, this theory can be criticized in more than one way but he holds that HABA «has not even put forward his criticism with the rigour required to be the object of a rational discussion", and asks HABA to do so.

Keywords: legal argumentation, E. P. HABA, M. ATIENZA.

* Fecha de recepción: 9 de mayo de 2011. Fecha de aceptación: 13 de mayo de 2011. 
1.

El título elegido para este comentario a la crítica feroz dirigida por E. HABA contra la «teoría estándar» de la argumentación jurídica [«Razones para no creer en la actual Teoría (ilusionista) de la Argumentación...»] es una broma que, estoy seguro, mi amigo (y crítico), autor de la misma, no se tomará a mal. Como el lector (por lo menos, el lector de cierta edad) habrá advertido, tomo el título de una de las obras de MARX (y ENGELS; aunque el papel de este último parece haber sido escaso), de 1844: La Sagrada familia. Crítica de la crítica crítica. Contra Bruno Bauer y consortes. Si bien es cierto que las posiciones iusfilosóficas de HABA están lejos del neohegelianismo de BAUER (y otros autores de menor relieve a los que despectivamente el mordaz MARX califica de «consortes»), me parece que hay algo en su afán crítico (superabundantemente presente ya en el título de su trabajo) que le emparenta con aquellos hipercríticos (críticos críticos). O sea, la de HABA no pretende ser una simple crítica, una crítica más; sino una crítica muy crítica: una crítica crítica, vaya.

2. Al igual que los profesores que repiten una y otra vez lo que consideran son las ideas fundamentales sobre un cierto tema, ante el temor de que sus estudiantes no les hayan entendido bien —o simplemente con el propósito de que, al menos, se hayan enterado de eso, de lo fundamental—, en su descalificatoria crítica de la «teoría estándar» de la argumentación jurídica, HABA repite machaconamente su mensaje, su crítica que, en lo esencial, parece reducirse a las tres tesis siguientes: 1) los argumentativistas incurren en el «síndrome normativista»; 2) desvían su foco de atención de las argumentaciones jurídicas reales (se fijan en algunos aspectos semánticos pero dejan fuera las dimensiones pragmáticas), y 3), como consecuencia de todo ello, lo que construyen es un discurso ideológico dirigido a justificar el razonamiento tradicional de los juristas.

Con lo de «síndrome normativista», HABA quiere decir que el conjunto de autores que integran la «teoría estándar» (un conjunto, por cierto, que en su texto no está muy claramente especificado; sobre esto volveré luego) se desentiende de una serie de vicios de razonamientos, de falacias que se encuentran corrientemente en los discursos de los profesionales del Derecho (lo que permite, en consecuencia, una presentación «embellecedora» de esos discursos). Tales vicios consistirían fundamentalmente en: poner el énfasis en las controversias terminológicas; recurrir a un «platonicismo normativista» que posterga el plano de la realidad; incurrir en diversas peticiones de principio; usar una terminología esencialista; no distinguir «es» y «debe»; recurrir a conceptos jurídicos indeterminados como si remitieran a un solo sentido identificable; usar fórmulas vacías persuasivas («proporcionalidad», «equilibrio», «racionalidad», «razonabilidad»); recurrir a nociones del pensamiento social vulgar («justicia», equidad», «bien común») sin ninguna precaución analítica.

La segunda de las críticas viene a consistir en que los argumentativistas no han construido una teoría realista de la argumentación jurídica. Construyen un modelo de la argumentación jurídica idealizado y que no se corresponde con cómo se argumenta realmente en el Derecho, con lo que ocurre en la práctica judicial y administrativa. La reconstrucción de la argumentación jurídica que los autores de la «teoría estándar» llevan a cabo, en definitiva, no es un retrato fiel de la realidad, sino más bien el resultado de haberla sometido a una operación de «cirugía estética». 
Y, en fin, la acusación de ideología (implícita ya en las anteriores críticas) no se debe a que HABA niegue que los ideales puedan jugar algún papel en el pensamiento social. Sobre esto, HABA distingue cuatro modelos de aproximación a las cuestiones sociales que apelan a lo ideal: el modelo de ideal valorativo (ideal querría decir algo que se juzga deseable y bastante viable); el modelo como «tipo ideal» (en el sentido de M. WeBER); el modelo de tipo promedial; y el modelo de idealización (que disimula y «embellece» cómo son ciertas realidades sociales). El carácter ideológico de la teoría estándar consistiría en que la misma: ofrece una perspectiva que es principalmente axiológica, pero de modo implícito hace creer que se trata de una descripción empírico-promedial; el modelo de argumentación jurídica que propone la teoría no es un tipo ideal en el sentido weberiano, pues con él no se pretende comprobar si las argumentaciones reales se apartan o no del mismo (o sea, no es un modelo descriptivo-crítico); se trata fundamentalmente de un modelo de idealización que encubre la realidad (digamos, de una ideología más o menos en el sentido marxista de la expresión).

3. Yo creo que HABA tiene alguna razón en lo que dice, pero no en cómo lo dice ni tampoco (y sobre todo) en la consecuencia que pretende extraer de su crítica: la descalificación radical de la teoría estándar de la argumentación jurídica.

3.1. Cuando digo que HABA tiene algo de razón, me refiero a lo siguiente. Hace unos veinte años, en una obra dirigida a exponer las teorías de la argumentación jurídica del siglo XX, dirigí a la «teoría estándar de la argumentación jurídica» (creo haber sido el inventor del término) una serie de críticas que tienen más de un punto de contacto con las de HABA. En efecto, en el capítulo final de esa obra ${ }^{1}$ señalaba que una teoría de la argumentación jurídica debía evaluarse desde tres perspectivas distintas, esto es, considerando cuál era el objeto, el método y la función de la misma. La «teoría estándar» (bajo este rótulo analizaba las de ALEXY y MACCORMICK, pero consideraba que también lo escrito por otros autores como AARNIO, PECZENIK o WRÓBLEWSKI tenía un significado semejante) presentaba, en mi opinión, tres tipos de insuficiencias. Desde el punto de vista del objeto, señalaba las siguientes: deja fuera las cuestiones de tipo fáctico las argumentaciones sobre hechos; tampoco se ocupa de la argumentación en el ámbito de la producción del Derecho; sólo considera el proceso de la «adjudicación» y no otros medios «alternativos» (pero también —o, al menos, parcialmente— jurídicos) de resolución de disputas, como la mediación, el arbitraje o la negociación; atiende exclusivamente al contexto de justificación (aunque no dé cuenta completa del mismo) y no también al contexto de descubrimiento. En cuanto a la metodología, mis críticas se centraban en dos puntos: 1) la teoría estándar no había elaborado un procedimiento que permitiera representar adecuadamente cómo los juristas fundamentan de hecho sus decisiones; 2) los criterios de racionalidad práctica construidos para juzgar acerca de la corrección de las argumentaciones son sólo criterios mínimos y de ahí que su utilidad sea limitada. Finalmente, a propósito de las funciones que debería cumplir una teoría de la argumentación jurídica, distinguía entre funciones teóricas o cognoscitivas, prácticas o técnicas y políticas o morales. A este respecto, señalaba diversos déficits de la teoría estándar y mostraba cómo los representantes de la misma propendían a una

${ }^{1}$ M. ATIEnZA, Las razones del Derecho. Teorías de la argumentación jurídica, 1. ${ }^{a}$ ed., Madrid, Centro de Estudios Constitucionales, 1991. 
valoración excesivamente positiva de los Derechos modernos y de la práctica de su interpretación y aplicación.

Por lo que hace específicamente al caso de ALEXY (autor al que HABA se refiere — por así decirlo — con una especial saña), pensaba (y sigo pensando) que su obra era (es) de una extraordinaria importancia: un hito en el desarrollo de las teorías de la argumentación jurídica. A pesar de lo cual, en aquel libro le dirigía algunas críticas: unas tomadas de otros autores (como NEUMANN, GIANFORMAGGIO o el propio HABERMAS), y otras de cosecha propia. Dado que lo que sostenía (y sostiene) ALEXY, esencialmente, es que la argumentación jurídica, en todas sus instancias, es un caso especial de lo que llama el «discurso práctico general», esto es, el discurso racional, dividía dichas críticas en dos grupos, según las mismas apuntaran a la teoría del discurso en cuanto tal o bien, específicamente, a la tesis del caso especial. En este segundo grupo de críticas distinguía todavía entre: una crítica conceptual, una crítica centrada en el alcance práctico de la teoría, y una crítica ideológica. 1) La de carácter conceptual se refería tanto a ciertas ambigüedades que cabía encontrar en la obra de ALEXY (por ejemplo, a propósito de lo que debe entenderse por «argumentación jurídica» o «discurso jurídico») como, sobre todo, al alcance de la noción de «pretensión de corrección». En relación con esto último, me parecía que podían surgir dudas, por ejemplo, acerca de si la pretensión podía plantearse también a propósito de las argumentaciones que llevan a cabo las partes en un proceso o incluso de las argumentaciones de los jueces y de la dogmática, puesto que las mismas tienen que moverse dentro de los límites del Derecho positivo, el cual puede incluir normas injustas. 2) En cuanto al alcance de la teoría, no veía tampoco que el modelo de ALEXY permitiera integrar adecuadamente la racionalidad discursiva con elementos de racionalidad estratégica, y los criterios que ALEXY sugería para medir la racionalidad de las decisiones jurídicas me parecían, al mismo tiempo, excesivamente latos (dado su carácter esencialmente formal) y excesivamente estrictos (consideraba más bien dudoso que muchas de las reglas del discurso racional se aplicasen en los diversos contextos de la argumentación jurídica). 3) Finalmente, la crítica ideológica se refería al riesgo de que la teoría pudiera contribuir a justificar de manera acrítica un determinado modelo de Derecho: el del Estado democrático y constitucional. Esos riesgos ideológicos derivaban: a) de que si bien la exposición de las reglas del discurso práctico general se hacía desde una perspectiva inequívocamente prescriptiva, cuando se pasaba al discurso jurídico se volvía esencialmente descriptiva: las reglas de la razón jurídica venían a ser las tradicionales del método jurídico; b) de la idealización de algunas de las instituciones centrales del Derecho moderno, como la dogmática o el proceso; c) de que ALEXY no parecía tomar en consideración la posible existencia de casos trágicos en el Derecho, casos para los que no existiría ninguna respuesta correcta; $d$ ) de que la defensa de una conexión necesaria entre el Derecho y la moral tenía el riesgo de atribuir a lo jurídico un sentido encomiástico en una forma que podría resultar arbitraria.

En fechas más recientes he vuelto a ocuparme de la concepción de la argumentación jurídica de AleXY y, en particular (junto con J. RuIZ MANERO), de su teoría de los principios. Creo que mi visión del Derecho es esencialmente coincidente con la suya, pero la tesis del caso especial tiene para mí el inconveniente fundamental de que uniformiza demasiado la argumentación jurídica, lo que supone, a su vez, el peligro de una 
teoría no suficientemente articulada (en el plano conceptual o analítico), menos útil en la práctica de lo que debiera ser (por su falta de realismo, de potencia descriptiva) y proclive a presentar la práctica jurídica (o algunos aspectos de la misma) en forma algo ideológica (dada su tendencia a la idealización). Yo no creo que, en rigor, la argumentación jurídica (si bajo este concepto se incluye no sólo la argumentación de los jueces y de la dogmática, sino también, por ejemplo, la de los legisladores y abogados) sea un caso especial de la argumentación práctica general. La argumentación jurídica (entendida en ese sentido amplio) es una práctica compleja en la que concurren diversos tipos de argumentación, de diálogos, y en donde, dependiendo del contexto de que se trate, puede prevalecer uno u otro de esos tipos de argumentación. Por ejemplo, parece evidente que los componentes retóricos tienen una extraordinaria importancia (constituyen quizás el elemento predominante) en la argumentación de los abogados (o en alguno de los contextos de la argumentación forense), mientras que no ocurre lo mismo con la argumentación de los jueces dirigida a justificar una decisión. Me parece que cuando se trata de, a partir de todas esas prácticas, construir algo así como una teoría general de la argumentación jurídica, debe dársele cierta prioridad al discurso práctico racional (al diálogo crítico); o sea, las formas estratégicas de argumentación no están a la par de la argumentación crítica racional. Pero para lograr eso, esa prevalencia del discurso práctico racional, basta con que el diálogo racional permita justificar (como creo que lo permite) la existencia de esas otras formas de argumentación: el discurso predominantemente estratégico de los abogados, los legisladores, los negociadores... O sea, yo no creo (como ALEXY) que las reglas del discurso práctico racional definan una especie de superjuego que contiene — en forma muy abstracta - a todos los otros juegos argumentativos (jurídico-argumentativos); y me parece que es relativamente fácil mostrar que existen ámbitos de la argumentación jurídica (por ejemplo, la del abogado que negocia un acuerdo con la otra parte) en las que no rigen (o no del todo) las reglas de la argumentación racional de ALEXY.

3.2. Cuando digo que HABA no tiene razón en cómo expone sus críticas (en las que, insisto, tiene algo de razón) no me refiero solamente a cuestiones que pudieran llamarse estilísticas. Lo que quiero decir es que su método crítico (o crítico crítico) es equivocado: sus críticas están mal dirigidas (equivoca el blanco) y están mal formuladas, en el sentido de que incurre en diversas falacias. Me explicaré.

Si están mal dirigidas es porque parecen dirigirse contra una serie de autores, de manera bastante indiferenciada, cuando resulta que entre ellos hay diferencias de cierta importancia. A eso es a lo que me refería cuando antes apuntaba al uso poco claro que HABA hace de la expresión «teoría estándar de la argumentación jurídica». Por ejemplo, HABA no parece incluir aquí a MACCORMICK (o no es claro que lo haga: las referencias al mismo son indirectas), que sería un candidato «natural» a semejante rótulo. Pero, sin embargo, sí que incluye a FERRAJOLI lo que, en mi opinión, resulta absolutamente gratuito. FERRAJOLI tiene una concepción del Derecho bastante distinta a la de autores como ALEXY, MACCORMICK o DWORKIN: aquél (a diferencia de éstos) es un firme partidario del positivismo jurídico; es un relativista y no cognoscitivista en materia de ética; y su enfoque del Derecho, simplemente, no es argumentativo (al contrario, una crítica que se le puede hacer — que se le ha hecho- es que descuida ese aspecto en su teorización del Derecho). Y, en fin, por lo que a mí respecta, los repro- 
ches que parece dirigirme HABA (al incluirme dentro de los partidarios de la «teoría» estándar) carecen simplemente de sentido. Obviamente, no porque yo crea que lo que he escrito sobre argumentación jurídica es insusceptible de crítica (de crítica fundada), sino porque la de HABA yerra manifiestamente el tiro. Aunque en muchos aspectos (seguramente, en lo esencial) coincida con ALEXY o con MACCORMICK (más con el último que con el primer MACCORMICK), ya antes he señalado que mi actitud frente a esos autores nunca ha sido la de aceptar sin más sus tesis y que las críticas que les he formulado desde hace tiempo se solapan parcialmente con las de HABA. Pero es que, además, el trabajo sobre argumentación jurídica que he efectuado en los últimos tiempos va exactamente en el sentido opuesto al imaginado por HABA: he efectuado muchísimo trabajo de «campo» dedicado al estudio de argumentaciones concretas (de las argumentaciones reales llevadas a cabo por los juristas) y me he interesado, de manera muy particular, por el estudio de las falacias, de los malos argumentos que tienen la apariencia de ser buenos; sobre ello he publicado un libro que contiene el análisis de cerca de un centenar de falacias extraídas de la discusión en los medios de comunicación españoles a propósito de diversas cuestiones de naturaleza jurídica, moral y política ${ }^{2}$.

Esa familiaridad con las falacias, por cierto, me lleva a pensar que la crítica de HABA incurre en más de una. Quizás la más importante de todas sea la que podría denominarse «falacia de la indeterminación». Consiste en criticar una postura, una cierta teoría, eludiendo toda precisión. Así, en el caso de HABA, uno no sabe - no puede saber- quiénes son exactamente los autores y las tesis criticadas: en su trabajo no hay prácticamente ninguna cita precisa de lo que pretende criticar, sino más bien meras referencias indeterminadas a algún autor y a su obra; e incluso la clase de los autores que integrarían la «teoría estándar», el objetivo de su crítica, está, como acabo de señalar, mal construida. Otra falacia en la que incurre con profusión es la que alguna vez he llamado «falacia de la exageración»: consiste en radicalizar una verdad, extenderla fuera del campo en que resulta aceptable, lo que la vuelve falsa. Así, es cierto que autores como AleXY, MCCormick, AARNio o PECZENIK, por lo que yo sé, no se han interesado por los argumentos falaces, pero es una exageración sin ningún fundamento pensar que, por ello, incurrirían en lo que HABA llama el «síndrome normativista»; a lo que se refiere $\mathrm{HABA}$ con ello es a una serie de consideraciones que cualquier autor con formación analítica (como los mencionados) conoce bien y no hay ninguna razón para pensar que se desentiendan — como HABA lo afirma- de esos vicios de razonamiento. Es más, HABA los acusa (en mi opinión con ningún o muy poco fundamento) de idealizar, de «embellecer» la realidad social, pero lo que el propio HABA hace con ellos, con sus teorías, es una operación semejante, pero de signo contrario: distorsiona esas teorías, las «afea» hasta un punto en que resultan irreconocibles (y, claro está, fácilmente - pero también inocuamente- criticables); o sea, HABA incurre en el vicio de la idealización, pero no con un afán de embellecer la realidad, sino de afearla. En fin, un rasgo del escrito de HABA que no sé si se puede calificar como falacia pero que, desde luego, es un caldo de cultivo propicio para cometer falacias, es lo que no dudo en llamar el «síndrome del crítico»: consiste en un empeño, en una obsesión, por asumir el punto de vista más crítico posible sobre una determinada cuestión, de

2 M. Atienza, La guerra de las falacias, 3. ${ }^{a}$ ed., Alicante, Librería Compás, 2009; también Bioética, Derecho y argumentación (cap. I), Lima-Bogotá, Palestra-Temis, 2004. 
tal manera que cualquier otra posición ha de resultar insuficientemente crítica; dicho de otra manera, el abandono de una actitud simplemente crítica en favor de una más crítica (de una crítica crítica).

3.3. Como consecuencia de lo anterior, las pretensiones de HABA de haber efectuado una crítica radical de la teoría estándar de la argumentación jurídica me parecen simplemente infundadas. Y como yo no deseo cometer ninguna falacia, aclaro que lo que quiero decir con lo anterior no es que esa teoría, la teoría estándar, esté plenamente justificada, que no ofrezca ningún flanco para la crítica (incluso para la crítica radical). Lo que quiero decir es que HABA no lo ha hecho. Que ni siquiera ha planteado sus críticas con el rigor exigible para ser sometidas a una discusión racional (y crítica). Y que lo único que me queda para terminar este escrito de contestación a su trabajo es pedirle que lo haga. 